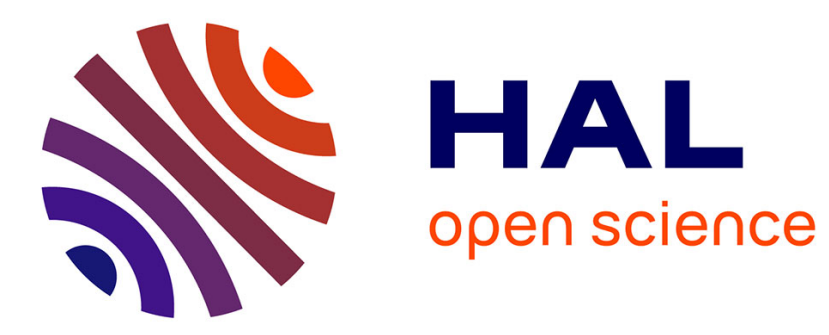

\title{
Face à la déprise : expériences de vieillissement et configurations d'aide formelle
}

\author{
Anastasia Meidani, Julie Bernard
}

\section{To cite this version:}

Anastasia Meidani, Julie Bernard. Face à la déprise: expériences de vieillissement et configurations d'aide formelle. Anastasia Meidani; Stefano Cavalli. Figures du vieillir et formes de déprise, Érès, pp.105-131, 2019, L’Âge et la vie, prendre soin des personnes âgées, 978-2-7492-6362-5. hal-02128703

\section{HAL Id: hal-02128703 \\ https://hal-univ-tlse2.archives-ouvertes.fr/hal-02128703}

Submitted on 14 May 2019

HAL is a multi-disciplinary open access archive for the deposit and dissemination of scientific research documents, whether they are published or not. The documents may come from teaching and research institutions in France or abroad, or from public or private research centers.
L'archive ouverte pluridisciplinaire HAL, est destinée au dépôt et à la diffusion de documents scientifiques de niveau recherche, publiés ou non, émanant des établissements d'enseignement et de recherche français ou étrangers, des laboratoires publics ou privés. 


\section{Figures du vieillir}

\section{et Formes de déprise}

Sous la direction de Anastasia Meidani Stefano Cavalli 


\section{Liste des auteurs}

ACETI Monica, Sociologue ; Lectrice au Domaine Sociologie, Politiques sociales et travail social, Université de Fribourg (Suisse).

ALESSANDRIN Arnaud, sociologue; Post-doctorant, Université de Bordeaux, Centre Émile Durkheim (France).

BERNARD Julie, Agent de service dans une résidence d'autonomie, Master Recherche en Sociologie, Université de Toulouse II Jean Jaurès (France).

BOUSIGUE Jean-Yves, Neuro-chirurgien ; Chercheur associé FRAMESPA (France).

CARADEC Vincent, Sociologue ; Professeur des Universités, Université de Lille 3, CéRIES (Centre de Recherche Individus Épreuves Sociétés) (France).

CAVALLI Stefano, Sociologue ; Professeur, Centre of Competence on Aging, University of Applied Sciences and Arts of Southern Switzerland (SUPSI) (Suisse).

CHAMAHIAN Aline, Sociologue ; Maîtresse de conférences en sociologie, Université de Lille 3, CéRIES (Centre de Recherche Individus Épreuves Sociétés) (France).

CHARPENTIER Michèle, Sociologue ; Professeure à l'École de travail social, Titulaire de la Chaire de recherche sur le vieillissement et la diversité citoyenne, Université du Québec à Montréal (UQAM) (Québec).

FERNANDEZ Guillaume, Sociologue ; Maître de conférences en sociologie, Université de Bretagne Occidentale, Labers (Laboratoire d'études et de recherche en sociologie) (France).

GAGNON Éric, Sociologue ; Enseignant-Chercheur, Université Laval, Centre de recherche sur les soins et services de première ligne, Centre d'excellence sur le Vieillissement (Québec).

GENTRIC Armelle, PU-PH gériatrie CHRU de Brest, Éthique, professionnalisme et santé - EPS EA 4686, UBO Brest (France).

GLENDENNING Jonathan, Sociologue; Doctorant, Université du Québec à Montréal (UQAM) (Québec).

GRIMMINGER Elke, Sociologue; Professeure des Universités à l'Institut de Sport et Science du Sport, Université Technique de Dortmund (Allemagne).

KNOBÉ Sandrine, Sociologue ; Ingénieure de recherche, laboratoire Sport et sciences sociales (E3SEA 1342), Université de Strasbourg (France).

LALIVE D’EPINAY Christian, Sociologue ; Professeur honoraire, Université de Genève (Suisse).

MEIDANI Anastasia, Sociologue ; Maîtresse de conférences en sociologie, Université de Toulouse Jean Jaurès, LISST (Laboratoire Interdisciplinaire Solidarités, Sociétés, Territoires) - CERS (Centre d'Étude des Rationalités et des Savoirs) (France).

PENNEC Simone, Sociologue; Maîtresse de conférences en sociologie, Université de Bretagne Occidentale, Labers (Laboratoire d'études et de recherche en sociologie) (France).

QUÉNIART Anne, Sociologue; Professeure des Universités, Université du Québec à Montréal (UQAM) (Québec).

VIEILLE MARCHISET Gilles, Sociologue; Professeur des Universités, Directeur du laboratoire Sport et sciences sociales (E3S-EA 1342), Université de Strasbourg (France). 


\section{Anastasia MEIDANI \& Stefano CAVALLI}

Introduction

Première Partie : Entre autonomie et dépendance, fragilité et vulnérabilité

Christian LALIVE D'EPINAY \& Stefano CAVALLI : Faire face aux perturbations de la vie : déprise et reprise au grand âge

Simone PENNEC : Déprise et trajectoires de santé : emprise, remise de soi et entreprise du souci d'autrui

Éric GAGNON : Le temps, les soins, la déprise

Julie BERNARD : Face à la déprise, la reprise : à propos de l'expérience des agents de service dans une résidence d'autonomie

Deuxième Partie : Parcours de santé et déprise : autour de la démence

Anastasia MEIDANI : Vivre avec la maladie d'Alzheimer en France, en Suède et en Grèce : Entre déprise, reprise et emprise

Guillaume FERNANDEZ : Entre continuité, déqualification, renégociation : quelles déprises après un diagnostic de maladie d'Alzheimer?

Vincent CARADEC et Aline CHAMAHIAN : Vieillir avec la maladie d'Alzheimer

Jean Yves BOUSIGUE : Sur la déprise : une expérience en neurochirurgie. Perspectives et retours d'expériences

Troisième Partie : Vieillir et se déprendre : une expérience corporelle genrée

Monica ACETI, Gilles VIEILLE MARCHISET, Sandrine KNOBÉ, Elke GRIMMINGER : Vieillir actif dans les programmes de promotion nationaux de la santé proposés en France, Suisse, Italie et Allemagne

Yves MORALES et Jean-Paul GENOLINI : Le réaménagement des activités physique au fil du temps

Michèle CHARPENTIER et Anne QUÉNIART (avec la collaboration de Jonathan 
GLENDENNING) : Vieillir au masculin. Entre déprise et emprise des normes de genre.

Arnaud ALESSANDRIN : Vieillir LGBT / Vieillir T. : la valeur heuristique du concept de la déprise 


\section{Face à la dé-prise : à propos de l'expérience des agents de service impliqués dans l'accompagnement des personnes vieillissantes}

\section{Anastasia Meidani et Julie Bernard}

Dans le cadre de cette contribution nous nous proposons de rendre compte des activités des aînés et des pratiques professionnelles du personnel impliqué dans son accompagnement sous l'angle de la théorie de la déprise. Il s'agira alors pour nous de développer une réflexion située au croisement des savoirs pratiques, de terrain et de la recherche, en lien avec cette même théorie, et en nous focalisant d'une part, sur les conditions de vie des personnes dites âgées, vivant en résidence d'autonomie ou à domicile et, d'autre part, sur les modalités de leur accompagnement et les mécanismes décisionnels situés à l'origine de leur placement en institution.

Trois angles d'approche seront retenus : le deuil de soi, le sentiment de solitude et la relation de l'aide. Du côté des soignés les plus âgés, il s'agira de rendre compte des formes de déprise observées au long de notre pratique professionnelle et de recherche et notamment de formes de reprise, de contrôle de pouvoir, de rebonds, de réorganisation de la vie, avec des stratégies d'adaptation observées dans (et en dehors de) l'espace de l'institution. Du côté des professionnels et dans une perspective interactionniste, nous ferons état de la manière dont les aidants représentent cette notion et la mettent en pratique, tout en explorant ses limites et les enjeux interprofessionnels qui l'animent.

L'article propose une illustration de mécanismes que Caradec (2004) déploie à propos de la déprise : l'adaptation (faire d'une autre manière), la substitution (faire autre chose de plus ou moins similaire), la délégation (demander de l'aide pour accomplir cette activité) et l'abandon (faire une autre activité plus adaptée à ses propres capacités). L'analyse proposée rend alors hommage aux travaux de l'équipe toulousaine : «c'est pour mieux "tenir" d'un côté qu'on "lâche" de l'autre. C'est un processus dont le rythme d'évolution est personnel, dont les formes particulières s'enracinent dans le monde antérieurement construit » (Clément et Mantovani, 1999, p. 5). Nous proposons d'analyser ce processus à travers trois dimensions constitutives de la théorie de la déprise perçue comme une série d'aménagement (Clément et Membrado, 2010). que nous exposons ci-dessous.

La question de la fin de vie est une problématique complexe (Gaucher et Van Lander, 2013) que le concept de déprise permet d'éclairer. Ce qu'il montre le mieux ce concept, c'est que le vieillissement et la fin de vie constituent une étape ultime de la construction du sujet qui pose la question du deuil de soi, tel que Déchaux (2002), nous l'a proposé avec le concept de «travail du trépas ». Ici la question du temps s'avère primordiale. À côté de cette première dimension de notre travail se dessine un autre axe qui concerne la solitude telle qu'elle est vécue et ressentie par les personnes vieillissantes elles-mêmes. Ce sentiment interroge l'expérience de solitude en situation de coprésence et certaines formes de la déprise (Campéon, Le Bihan, Mallon, 2012). Ici à travers les objets et le travail de réaménagement des activités quotidiennes susceptible de palier à ce sentiment, c'est le lien social qui se trouve interrogé. Enfin, un troisième point cible plus particulièrement la question de l'accompagnement du sujet en fin de vie. Cette dernière dimension questionne les configurations d'aide et amène à interroger la dynamique des échanges entres proches professionnels et aînés. Ces configurations d'aide se présentent comme une relation à construire qui est par ailleurs 
destinée à se rompre à court terme (Pitaud, 2013). S'attardant tour à tour sur chacun de ces aspects, ce texte cherche à montrer dans quelle mesure et de quelle manière le concept de la déprise est susceptible d'éclairer l'expérience du vieillir analysée du point de vue des professionnels impliqués dans leur accompagnement.

D'un point de vue méthodologique, l'article prend appui sur une série d'entretiens, formels et informels, réalisés dans le cadre de notre Master de Recherche en Sociologie et de notre travail en qualité d'agente de service dans les résidences d'autonomie ou à domicile. Les interviews réalisées se présentent alors comme le fruit de chaînes relationnelles et s'inscrivent dans l'analyse d'inspiration monographique d'un matériau ethnographique qui mobilise une expérience professionnelle de cinq ans. Plus précisément c'est en passant par ce poste professionnel, par des parents d'amis ou de la famille que les enquêtés ont été abordés. Pour la plupart, il s'agit donc des personnes de l'entourage professionnel, direct ou indirect, dont le recrutement a cherché à rendre compte de la pluralité des cas observés sur le terrain au long de notre parcours. Dans notre panel figurent : cinq personnes âgées de plus de 70 ans vivant en résidence pour l'autonomie, deux vivant à domicile et deux qui se sont rapprochées de leurs enfants. Pour ce qui est du personnel: quatre travaillent dans ces mêmes résidences, trois à domicile, une en milieu médicalisé et une en résidence pour l'autonomie et à domicile. Tous les entretiens ont été effectués sur la même base (un guide thématique pour les personnes vieillissantes et un autre pour les professionnels) et se sont déroulés à domicile sauf un (sur internet par skype). Ceux avec le personnel ont été conduits dans des lieux publics, sur les lieux de travail ou sur internet (par skype).

Les prises de contact se sont avérés simples et fluides, ces personnes nous connaissaient, ce qui a favorisé le déroulement des entretiens. En effet, elles remontaient, rapidement dans leurs souvenirs, les racontant, les revivant parfois avec une pointe de nostalgie que nous avons pue percevoir chez elles. Ce qui est particulier, c'est que même les souvenirs les plus douloureux se présentaient comme le symbole d'un passé qui valait la peine d'être vécu. Plusieurs enquêtés se sont laissés aller à des larmes, des éclats de rire ou des silences lourds de sens. Ces moments se sont souvent révélés d'une vive émotion marquant ainsi la charge émotionnelle de l'élaboration de ces expériences du vieillir.

\section{Autour de la proximité de la mort : une temporalité plurielle}

La première chose qui interpelle à la lecture de nos entretiens c'est la place centrale qu'occupe la mort dans les récits de nos interviewés. Nous pensions qu'il n'y avait pas de mots pour dire la mort mais nous nous sommes rendue compte qu'on était plutôt dans le registre de l'inaudible (Meidani, $2018 \mathrm{~b}$; Clément, 1994). Ce qui ressort de ces récits est avant tout une sensation de fatigue accumulée au fil des années et un sentiment de lassitude qui dicte la proximité de la mort. Josée ${ }^{1}, 93$ ans, placée en institution, nous en parle : "Je peux partir ce soir ou demain, ça m'est égal. Tout m'est déjà arrivé, les cliniques, les opérations, j'ai tout fait... non, je n'ai pas peur. Je n'ai pas peur, mais je ne voudrais pas souffrir. » Bien souvent il en ressort que derrière l'appréhension de la mort (ou son déni), c'est de l'anxiété d'être seul dont il est question, l'inquiétude qu'il n'y ait « rien après », la crainte de souffrir,

\footnotetext{
${ }^{1}$ Tous les prénoms renvoient à des pseudonymes.
} 
ou encore d'être oublié... Ici la peur épouse la forme du néant, de l'inconnu, pire encore d' « une vie qui n'a servi à rien ». Avoir raté son existence sans avoir le droit à un nouvel essai : tel semble être le pire des scenarii. Certains imaginent un paradis, une réincarnation, un vide, un rien... Hélène, 85 ans, nous raconte :

Peur de la mort? Ce n'est pas le mot... Mais ça me fait froid dans le dos. Alors que mon mari disait avec une voisine déjà morte, qu'ils n'avaient pas peur de ça... même si elle la voyait arriver, me soutenait-elle. "Moi, je ne suis pas comme vous», je lui disais, et ça fait quatre ans qu'elle nous a quitté. Il faudrait être bien sûr de ce qui nous attend pour ne pas en avoir peur!

C'est surtout à partir du départ de l'autre que cette prise de conscience de la proximité de la mort s'opère et la déprise permet d'investiguer cette expérience dans ses composantes relationnelles. Anne, 89 ans, qui vit toujours à son domicile, nous confie :

Je m'ennuie des gens qui sont partis. Beaucoup. Je leur parle, ils me laissent là, ils ne me veulent pas. Un jour, j'étais en colère, j'ai perdu ma clef et ça m'a pris alors que je la cherchais et je les ai engueulés : "vous ne pouvez pas m'aider au lieu de rester là ? "Je me suis dit, je suis folle... [...] J'ai eu quand même dans ma vie le bonheur et le malheur aussi d'avoir deux hommes avec qui on s'entendait bien, tous les deux hein. Ça allait avec eux à tout point de vue... Avec mon second mari, on s'était dit tous les deux, on riait en disant qu'il retrouverait sa femme car il était veuf... qu'en mourant, on ne savait pas comment on se retrouverait, avec qui. Lui, sa femme, et moi mon mari. On parlait facilement de nos morts, on était libre pour ça. On sait bien qu'on peut mourir chaque jour, et que ça ne s'arrange pas avec les années qui passent.

Les personnes que nous avons eues l'occasion de rencontrer au long de notre parcours professionnel et de recherche se sentent en quelque sorte «en attente». Baptiste, 86 ans, veuf qui réside à son domicile, nous révèle : «Je n'ai peur ni de l'avenir, ni de la mort. L'avenir c'est demain, quant à la mort, elle est programmée depuis le jour de ma naissance. » Mais parfois les récits laissent place à une note de déprime. Certains ressassent les souvenirs d'hier en pensant qu'ils n'ont plus d'avenir et plus rien de « grandiose » à vivre, tout en cherchant à positiver : « Maintenant, on laisse la place aux jeunes... ». Combien de fois avons eu à écouter cette phrase lors de nos interventions...

Si la mort est un sujet omniprésent pour ces personnes, les professionnels et la famille ne semblent pas prêts à l'accompagner - notamment lors de lourdes pathologies ou handicaps. Christine, une collègue de 55 ans, nous révèle :

Avec la profession que j'ai ici, je vois que les employées ne sont pas forcément prêtes à la mort. C'est pourtant un travail de fond, accompagner la vieillesse, jusqu'au fond, il faut accompagner la mort. Moi ça ne me choque pas, la mort est beaucoup plus choquante dans un service de natalité. 
Josyane, 61 ans, quant à elle, place le maintien de l'autonomie au centre des enjeux du «bien mourir » et décrit la lourde tâche du personnel dans ce travail de l'accompagnement de la mort (Ennuyer, 2014), complétant à son tour les propos de sa camarade :

J'aime mon travail car j'aime le contact avec les gens que je considère comme des clients. Grâce à eux, j'ai un travail et grâce à nous, ils peuvent continuer à vivre aussi confortablement que possible. J'ai pris beaucoup de recul vis-à-vis de la mort. Ça fait partie du boulot, même s'il n'y a pas d'indifférence, mais il faut gérer et être efficace. Il faut laisser le temps au temps [...] Pour moi [l'enjeu], c'est les maintenir le plus longtemps possible avec de l'autonomie, de l'aide mais pas d'assistanat, ne pas infantiliser les gens. Et ne jamais oublier avant d'être ce qu'ils sont maintenant avec leur pathologie, c'étaient certainement des gens formidables, il faut les imaginer jeunes et c'est comme ça qu'on arrive à les respecter, même avec une pathologie Alzheimer ou Parkinson.

D'autres encore insistent sur le support relationnel constitutif de leur métier à l'image de Christine, 55 ans, qui nous précise :

Je pense que c'est un métier très relationnel. Les résidents peuvent venir me trouver sans problème, ils parlent de leur vie, ils ont besoin d'être écoutés, surtout en fin de vie. En fin de vie, ils ont besoin de libérer tout un tas de choses qu'ils ressassent, des non-dits à la famille. J'ai toujours dit au personnel de discuter et les résidents sont contents de leur relation avec le personnel. Mais s'il y a une tension dans le personnel, ils le sentent et ils me le disent, ce sont des éponges.

Du point de vue des aînés, cette conscience d'être «au bout du chemin » active une gestion paradoxale de la temporalité. Il faut se dépêcher de "profiter» de ce et ceux qu'on aime et, à la fois, «savoir prendre le temps ». Mais de toute évidence, ce temps ne prend sens que devant la proximité de la disparition prochaine : la mort de soi, la mort de l'autre (Mouysset, 2017). Louis, 97 ans entré en institution depuis la mort de son épouse, nous décrit ainsi son expérience :

C'est dur d'accepter d'être tout seul, si vous avez des gens que vous aimez, aimez les fort et vite car ils disparaissent et ça, c'est dur. 90, 95\% sont des veufs ici... Moi ça va faire quatre mois et demi [il se réfère à la perte de sa femme] et c'est dur à accepter, on se connaissait depuis nos dix ans, c'était nos soixante-douze ans de mariage, on avait fait une belle fête. Faut se faire à l'idée qu'on n'est pas immortels, mais la directrice m'a bien aidé, elle a été présente psychologiquement pour accepter... faut se faire une raison.

Si le récit de Louis est significatif des négociations de soi avec soi lors de cette dernière étape de la vie, la gestion du temps n'a pas toujours été la même. Pour la majorité des aînés rencontrés, accéder à la retraite, c'est le moyen de " profiter » de sa famille, de son temps libre, et l'occasion de s'investir 
dans de nouvelles activités choisies (gymnastique, peinture...) ou à des clubs (jeux de sociétés, repas, sorties, petits voyages...). Baptiste, 86 ans, témoigne : "Comme activité principale, je fais des modèles réduits, des miniatures, ce qui me prend pas mal de temps. La lecture aussi, beaucoup, et un peu mon ordinateur. Depuis que je suis à la retraite, le temps me semble passer plus vite... »Claude, 86 ans, placé en institution, complète à sa manière :

[Mes activités] sont multiples et variées. L'ordinateur, j'ai repris en 2010 quand ma femme est décédée. La télévision, la musique, la médiathèque, mais la médiathèque ne prête pas les DVD car il faut les garder 15 jours maximum mais ils ne passent que tous les mois, ils vont peut-être arranger ça... On joue tous les soirs après les animations, les débats aussi. S’il y a de la gym j'y vais, ou une animation, l'après-midi...

En somme c'est se faire plaisir sans les contraintes d'un travail, des horaires et des missions. La fatigue n'est plus la même, la manière de faire les choses change aussi. C'est l'attention soutenue à l'égard du corps qui donne le temps esquissant en filigrane la logique anticipatoire de la déprise, ou pour ainsi dire la reprise (Clément, 2003). Bernard, 83 ans, vit toujours à son domicile. Il décrit ainsi son rapport à la vie ou, pour ainsi dire, son rapport avec la mort :

Personnellement, je n'ai pas de craintes, je ne suis pas du genre à m'affoler pour des choses comme ça. À 83 ans, on a déjà bien traversé la vie. On verra bien ce qui se passera. Je fais par contre un peu plus attention, à la façon dont je me tiens, à ne pas trop forcer dans certains cas pour m'éviter des douleurs dans le dos, ou lorsqu'on part quelque part, on ferme bien partout les volets pour éviter les cambriolages, des petites choses comme ça...

L'extrait donne un aperçu saisissant de cette logique d'accommodation, chère à la déprise (Clément et Mantovani, 1999).

\section{Se sentir seul en étant entouré : vie en institution et dé-prise relationnelle}

Au sein de l'institution les contacts sont fréquents avec le personnel : chaque jour pour certains, plusieurs fois par semaine pour d'autres. Les personnes qui coexistent dans un même espace sont amenées sinon fortement incitées à se côtoyer et à créer des liens les unes avec les autres. L'animation vient rythmer le temps pour qu'il devienne du temps à vivre une autre manière de lutter contre la froideur de l'institution (Vercauteren, Vercauteren et Chapeleau, 1993). L'animation cherche alors à maintenir le désir, désir de vivre, de partager, de participer... En ce sens et du point de vue des professionnels, elle désigne un indicateur de la qualité de la vie incontournable. Mais quel est le but recherché à travers ces manifestations ? Pour Christine, 55 ans :

Les animations jouent un vrai rôle dans le quotidien des résidents. Je peux le comparer car dans les deux établissements où j'étais avant, il n'y avait pas d'animation. Et il y avait 
beaucoup plus d'agressivité. Là, ils sont impliqués tous les jours. Il y en a pour tout le monde, à l'intérieur, à l'extérieur, des gens de l'extérieur viennent, quand il le peut le personnel participe. Ça égaye véritablement nos résidents, ça permet une bonne ambiance et qu'ils se côtoient encore davantage en dehors des repas.

Au-delà des manifestations, d'autres moyens sont ici mobilisés pour soutenir la participation à la vie de la communauté. Le «projet de vie » en est un (Vercauteren, Hervy et Schaff, 2008) et les travaux sur la déprise permettent d'en rendre compte (Meidani, Membrado, 2011). Claude, 86 ans commente ainsi ce dispositif: "Des projets ? Bien sûr. Est-ce qu'ils sont réalisables, je ne sais pas. J'ai une nièce qui va se marier, j'aimerai y aller. Un neveu aussi, plus tard... » A contrario, Anne, 89 ans se montre beaucoup plus critique :

Que voulez-vous que j'ai comme projets? J'en ai pas c'est ça qui me manque. Je dis que j'aimerais faire ci ou ça, mais avec quoi, avec qui ? Pour les voyages je referai plus, s'il y avait des amis de l'extérieur qui viennent avec moi oui, mais ici, il n'y a plus personne...

$\mathrm{Si}$, en principe, en institution les résidents sont libres de choisir parmi les options proposées (aller ou non aux sorties, prendre ou non les repas), elles doivent tout de même se plier aux règles communes de l'établissement, aux horaires, à l'organisation, aux transports. Pour celles qui jouent le jeu, c'est le moyen d'avoir une vie à la fois divertissante, remplie et ordonnée. C'est bien ce qui incite Louis, 97 ans, à comparer ce cadre de vie à des « colonies de vacances » :

On n'a pas beaucoup d'obligations ici. Peut-être les horaires, mais c'est normal dans une collectivité, pour les repas, il faut être à l'heure... J'ai fait des colonies de vacances en tant que moniteur et directeur pendant une quinzaine d'années, il fallait des règles là aussi [...] Moi je ne descends pas tous les jours aux animations, mais Claude [un autre résident] oui, tous les jours, de 17 hà 18 h30 pour le Triomino. Quand ça m'intéresse j'y vais, sinon non. Hier avec les petits enfants, non, ça va, j'ai assez donné... Je suis actif mais pas entièrement avec les collègues de la résidence. Quand on était une cinquante, je connaissais tous les noms, mais à cent, je ne peux pas, on est trop, on ne peut pas se lier à tout le monde...

La fin de l'extrait montre bien la difficulté d'encourager le lien, dès lors que les institutions d'accueil ressembler à des « usines », une critique qui revient souvent dans la bouche de ces aînés.

L'entretien du lien avec les autres résidents ne semble pas une simple affaire. Anne, 89 ans, nous confie :

J'ai quand même eu une dispute. Ça allait à peu près et puis elle [une autre résidente] me faisait beaucoup de remarques et un soir on jouait ensemble avec un autre monsieur, elle s'était plaint de ce monsieur. Moi, il m'allait très bien tant qu'il était correct. Et puis un autre soir, alors 
qu'on cuisinait, elle m'a attrapée et m'a dit: "Toi tu sais, tu cherches les hommes quand même, tu t'assoies toujours à côté d'Alfred. "Je n'ai pas répondu, et à la fin, elle me dit : "Et Clarence [une autre résidente] m'a dit des choses sur toi ». Ça m'a fait vraiment mal, car le reste je m'en fichais car ça ne la regardait pas et j'avais ma conscience... [...] Le lendemain, je ne suis pas descendue ni rien, elle a vu que je faisais la tête, j'étais pas venue faire de gâteaux et ça s'était arrêté là. Mais elle est revenue me voir et je lui ai dit que notre amitié était finie, car je l'ai mal pris, ça m'a fait mal. Je me suis un peu isolée, retirée de la vie commune depuis.

De nombreuses histoires de ce genre nous ont été livrées. Mais dans leur majorité, les membres $\mathrm{du}$ personnel ne partagent pas forcément ces interprétations (Mauriat et al., 2010). Beaucoup nous parlent des "personnes âgées caractérielles qui se mettent elles-mêmes en situation d'échec vis-à-vis des autres », considérant qu'en foyer logement la vie en communauté est censée faciliter les échanges entre résidents. Christine, 55 ans, commente à ce propos :

Celui qui va s'exclure, c'est celui qui va générer lui-même l'exclusion, un mauvais caractère... Quand on donne de la colère, la haine... ça isole. Quand ils viennent me voir pour s'en plaindre, je leur dis que c'est de leur faute. Ils l'entendent ou ils ne l'entendent pas, au moins, c'est dit. Mais c'est rare, sur cent personnes on doit en avoir cinq, six au plus.

Cherchant à tenir à distance toute histoire de ce genre qui viendrait noircir le tableau, le personnel de service force le trait de cet art de relationner constitutif de son métier. En essayant de légitimer l'interventionnisme du personnel dans la sphère de l'intime, les professionnels se plaisent de répéter que bien souvent ils constituent la «seconde famille pour les personnes âgées ». Eliane, 63 ans, commente :

Je suis un repère sécurisant dans la mesure où je prends des initiatives et c'est comme si je faisais partie de la famille. Je les connais depuis trente ans. Je prends les devants quand je vois leurs besoins et les autres professionnelles me demandent conseil à de nombreuses occasions.

Lucie, 59 ans, abonde dans cette même direction :

Je pense représenter une sorte d'ange-gardien pour les personnes âgées, j'assure leur hygiène, et même si c'est parfois dur pour elles, c'est extrêmement important. Je ne fais pas que les soigner et les laver, je les rassure et les réconforte.

Josyane à son tour enfonce le clou :

Je n'ai pas cette vision de la vieillesse car il n'y a pas beaucoup de différence d'âge entre eux et moi. Ce serait peut-être différent si j'avais vingt ou trente ans de moins. Il y a des jeunes qu'on 
a eu en stage ou formation, la personne âgée était rébarbative et ils étaient rebutés par les visages ridés ou autres et c'est compréhensible. Quand on arrive à 60 ans, on voit ça différemment...

Mais Josyane, Eliane et Lucie ont près ou plus de 60 ans. Dans ce sens, ce que laisse entendre leur témoignage, est un écart générationnel assez net, observé à maintes reprises durant l'exercice de nos fonctions, où notre «jeune» âge a souvent fait l'objet de commentaires, plus ou moins désobligeants, de la part de nos collègues mais aussi des personnes auprès desquelles nous avons eu à intervenir. Ernestine, 89 ans, placée en institution, confirme :

Il faudrait mettre les petites jeunettes au pli quand même. Il faut voir comment elles sont des fois... Pas arrogantes mais, elles manquent d'aide pour savoir comme faire avec les personnes. Avant, ce n'était pas la même mentalité, elles sont trop brutes, personne n'est parfait mais je vois les jeunes, faut les guider. [...] il leur faudrait une formation ici, c'est un métier qui s'apprend, parfois elles ne sont pas douées... certaines viennent à quarante ans et on voit qu'elles sont mieux pour aider, plus d'expérience, elles savent y faire et puis ce n'est pas la même éducation.

À l'instar de Christine (dont les témoignages ont été présentés plus haut), généralement, les professionnels ont du mal à comprendre le ressenti de solitude qu'ils vivent plutôt comme une remise en cause de leur travail. Patricia, 42 ans, décrit les choses ainsi : "J'entends tous les jours des personnes âgées me dire qu'elles se sentent seules... j'ai du mal à comprendre car elles sont entourées, famille, soignants, résidents... Je pense que je comprendrai lorsque je serai âgée et dans une structure. » Et pourtant, les propos de cette résidente Josée, 93 ans, est explicite et donne le ton dès le début de notre entretien :

C'est la solitude ici... Je ne vais pas vous dire des choses très agréables... j'ai du mal à m'adapter. Il y a beaucoup de personnes, c'est chacun pour soi, personne ne se dérangera pour vous. J'ai fait des connaissances, avec celles qui sont agréables, mais c'est tout. Il y a beaucoup de racontars, alors je ne dis plus rien car ça cause des ennuis. J'ai quelques amies ici, on discute un peu. À l'extérieur j'en ai quelques-unes mais elles sont loin, on s'écrivait mais à notre âge c'est difficile de garder le contact. Mes petits enfants viennent me faire les courses tous les quinze jours, ou ils me téléphonent souvent. Je les adore, ça me fait plaisir. Je me sens seule, mais isolée non, c'est mon caractère qui veut ça, j'ai l'habitude. J'arrive pas à $m$ 'adapter, d'autres qui sont arrivées récemment sont déjà chez elles... moi, je ne suis pas chez moi, même si je n'ai rien à redire.

En guise de conclusion de cette partie qui a insisté sur la place de l'autre dans les dé-prises relationnelles en institution, il conviendrait d'apporter une précision qui nous parait capitale : la participation des professionnels à la coproduction de la déprise sous son versant relationnel ne se réduit pas à la vie en communauté. Le personnel qui intervient à domicile représente aussi un ressort relationnel précieux pour les personnes vieillissantes. Céleste, 83 ans, l'affirme clairement : «Je parle 
beaucoup avec ma femme de ménage, on discute de sa fille, de mes enfants et mes petits-enfants et mon arrière-petite-fille aussi. De sujets plus bateau aussi... C'est agréable d'avoir une personne extérieure à la maison pour parler. »

\section{Entre accompagnement et placement : la présence de l'autre dans la dé-prise}

En général, le placement en institution n'est pas vécu comme un choix, mais une nécessité qui marque un pas décisif dans le processus de déprise souvent accompagné d'une certaine souffrance (Donnio, 2005). Mais cette position peut évoluer au fil du temps attestant des efforts de la vieille personne de s'adapter au nouveau contexte. Le témoignage de cette professionnelle rend compte de la pluralité des cas rencontrés sur le terrain :

Certaines personnes disent "tant que je peux rester chez moi», et que les enfants mettent en place du portage de repas, une aide-ménagère, une infirmière et tant qu'elles arrivent à sortir un peu... Et d'autres disent qu'ils auraient dî venir avant, en étant encore un peu autonome, pour avoir moins de difficultés à s'intégrer. Reprendre une vie en communauté après avoir vécu seul ou en couple, ce n'est pas évident. Il y a des règles et des horaires, on mange avec des inconnus, et cette démarche, à 80 ans, ce n'est pas simple. Il faudrait dix ans de moins pour accepter ces contraintes. Un monsieur est parti comme ça, soit il était trop valide, soit il ne $s$ 'adaptait pas. Il est reparti dans un appartement, ça ne lui allait pas (Josyane, 61 ans)

Le choix de ce résident évoqué par Josyane montre bien que la décision n'est pas irréversible. Néanmoins de telles exceptions viennent confirmer la règle. Une fois le palier de l'institution franchi, la personne ne peut que rarement revenir en arrière.

Dans la majorité des cas, le processus décisionnel qui précède le placement correspond souvent à un temps de crise familiale intense ou se mélangent culpabilité, angoisse devant l'inconnu et souffrance liée au constat qu'un nouveau cap est franchi en direction de la fin prochaine (Gramain, Lacan, Weber et Wittwer, 2005). Pour les enfants, s'il est délicat de prendre la décision du placement, il est tout aussi délicat de «devenir le parent de ses parents». Pour gérer la charge du travail, la famille doit soustraire à la volonté de la personne ; et cette décision se prend au nom de «son bien » et de sa «sécurité ». Mais la décision implique un travail de «deuil» lourd de sens qui est vécu comme une épreuve.

Je crois que placer ses parents en institution est une grande souffrance pour les familles. Quand elles entreprennent les démarches, les secrétaires nous disent que ce n'est pas spontané, il faut du temps pour prendre la décision. Je ne pense pas que ce soit de gaité de cour pour la majorité, c'est compliqué pour les deux, il faut faire le deuil de beaucoup de choses. Elles ne peuvent pas faire autrement, elles pensent d'abord à la sécurité de leurs parents et leur bienêtre et elles s'y résolvent (Josyane, 61 ans) 
Au-delà du bien-être de la personne celui de la famille entre aussi en ligne de compte, alors que la solitude semble orienter aussi le choix opéré. Le sentiment d'«abandon » pointe souvent les mises en récits de ces résidents que ces professionnels n'oublient pas de relever.

Certaines personnes âgées voient cela comme un abandon de la part de la famille, mais c'est très souvent pour leur bien-être et aussi pour celui de la famille [...] Le placement d'une personne âgée est souvent fait après le décès du conjoint, car elle se retrouve seule chez elle. Je pense qu'en premier, c'est la solitude qui pousse à venir en institution, après arrive la sécurité, surtout si la personne âgée a des troubles du comportement, ou une maladie qui amène ces troubles. (Patricia, 42 ans)

Mais les décisions prises ne vont pas toujours dans le sens souhaité par les personnes qui, dans le meilleur de cas, finissent par se résigner à faire ce qui parait être «le plus adapté à leur âge et état de santé... ». Josée, 93 ans, rend compte ainsi de son expérience :

Vue ma situation, je ne pouvais plus continuer à vivre seule chez moi. C'était une obligation de venir ici, pas un choix. Je n'avais plus personne. Mes enfants sont décédés, mon mari aussi et moi je n'ai que deux petits-fils. Je ne pouvais pas rester toute seule dans mon appartement. [...] J'aime vivre ici... oui et non, je suis obligée... Mais on finit sûrement par s'adapter.

La fin de l'extrait de Josée, signe par excellence d'une tentative de prise, est à lire comme une antiphrase. En effet, dans ces cas de figure l'adaptation paraît compromise. Claude, 86 ans, esquisse ainsi les raisons qui ont motivé son placement en institution :

J'ai perdu ma femme, j'ai perdu en autonomie, et puis les enfants qui habitaient près de chez moi ont dî partir pour le travail... Tant que j'ai pu, je suis resté chez moi, mais quand je n'ai plus pu me déplacer correctement à cause de ma jambe, c'était pénible... Mes enfants m'ont installé ici pour me protéger. Quand je me suis retrouvé handicapé et que j'ai vu où ça allait, faut peser le pour et le contre.

Si sur ce point qui rend compte des raisons qui motivent l'entrée en institution, les avis des aidants et des personnes aidées convergent, d'autres sujets sont autrement polémiques. Dès lors c'est l'accompagnement, dans et en dehors des limites spatiales de l'institution, qui est questionné. Lors de nos échanges avec les professionnels au quotidien, nous avons souvent eu à entendre des commentaires, notamment de la part des plus jeunes de nos collègues, reléguant les personnes âgées dans le rang du «hors du coup», «en retard», «n'étant plus au fait des dernières avancées technologiques »(Caradec, 2001). Par ricochet, il se crée un véritable déni de la dynamique de la déprise dans son versant le plus positif : la prise. Et pourtant les personnes que nous avons eues à rencontrer qui tentent de comprendre et d'apprendre à se servir des nouveaux outils ne sont pas rares, 
même si certaines ont l'impression d'avoir un «train de retard sur la société ». Céleste, 83 ans, s'en amuse :

Moi, j'ai une tablette, oui! J'en avais envie parce que mon mari est très souvent à l'ordinateur! La tablette, c'est plus confortable pour moi! C'est un truc spécial pour les personnes âgées, plus facile, je n'ai qu'à appuyer sur ce que je veux faire avec, le scrabble, ou les recettes... et ma fille me montre ce que je ne sais pas faire. Je n'arrive pas pour les mails par contre...

Le témoignage de Céleste est à mettre en perspective avec les précisions apportées avant l'entretien où l'interviewée nous explique qu'elle vit dans une maison loin de tout, et que le premier village est à plusieurs kilomètres de chez elle. Comme elle ne conduit pas, Céleste est obligée de s'en remettre systématiquement à son mari, qui lui, conduit encore. Cependant, elle est consciente que s'il décède le premier, ou encore qu'il ne puisse plus conduire pour une raison quelconque, elle ne pourra plus vivre dans sa maison. La seule solution sera de se rendre dans une ville avec tout ce dont elle aura besoin à proximité (alimentation, docteur, pharmacie...). Voici comment Céleste rend compte de cet état de lieu :

Je suis complètement dépendante de mon mari pour sortir, les courses, le quotidien... Je n'ai pas le permis de conduire... S'il n'était plus là, il faudrait que je trouve quelqu'un d'autre, ou que je parte. C'est mon mari qui me permet de vivre ici, sans lui, je devrais déménager. S'il n'est plus là, j'irai près de ma fille.

L'expérience de Céleste garde à distance l'idée du placement en institution et exalte la dynamique de reprise. Mais son témoignage rend aussi compte des enjeux autour du lieu d'intervention de ces professionnels qui configure les modalités de participation du personnel à la mise en place des stratégies d'adaptation. Amenés à intervenir tantôt à domicile tantôt dans des établissements des soins, ces professionnels repèrent une distinction nette entre les deux espaces de vie. Eliane, 63 ans, professionnelle, nous raconte :

Cette maison est un cas très particulier parce que ces personnes ont de l'argent, ce qui leur permet d'avoir une bonne équipe pour leurs soins. Les enfants refusent qu'ils soient placés. Mais je suis certaine qu'ils sont mieux chez eux, ils gardent leurs repères et leur environnement. Ils ont leur rythme car nous nous sommes adaptés à leur vie et non l'inverse. Ils vivent selon leurs envies, leur liberté. Mais leur cas est très exceptionnel, nous sommes là 24h/24.

Quant aux aînés, et à leurs yeux, le domicile est représenté comme un précieux levier d'autonomie. Bernard, 83 ans, en atteste : 
S'il y avait des raisons valables, oui, on irait [en foyer logement, ou maison médicalisée selon leur état], s'il n'y a pas de possibilités de faire autrement. La question ne se pose pas... on a la volonté de rester ici, donc ça sera à contrecour pour moi. J'ai suffisamment visité des amis dans des maisons de retraites pour savoir que si on a toute sa tête, vivre là-dedans, c'est infernal... Il suffit de les voir dans la salle de télévision pour voir ce qui se passe dans le regard de ces gens, ça fait peur. Je me sens à l'abri chez moi, c'est mon cocon [...] Je regarde la télévision, j'écoute de la musique... un ordinateur pour les jeux aussi, jamais je ne m'ennuie. Je fais ce que je veux, je trouve toujours quelque chose à faire. Je bricole un peu quand ça me prend. J'ai une passion pour l'image alors je découpe beaucoup dans les revues les images.

Nous discernons ici la place qu'occupent les activités quotidiennes dans les tentatives du sujet de réserver un certain contrôle sur le sort de sa vie. Si les problèmes de santé viennent conditionner ces stratégies d'adaptation, les résistances ne manquent pas. De nombreuses personnes parmi celles que nous avons eues à rencontrer s'efforcent malgré leur fatigue et leur faiblesse à effectuer les tâches du quotidien. D'abord parce que c'est un «moyen pour elles de se tenir en forme », mais aussi parce que cela fait partie de la «routine ». Mais pas de n'importe quelle routine : la leur! Elles ont l'habitude de se faire la cuisine, de s'occuper de leur jardin et elles y tiennent pour protéger leurs « gô̂ts » et leurs "envies». Cette manière de s'accrocher au quotidien constitue un support hors pair d'autonomie. C'est en cela que cet ancrage de la déprise dans l'ordre du faire est crucial : car il décrit son attachement aux pratiques. Ces dernières sont souvent investies d'une question de dignité qui devient alors un moteur qui anime l'engagement de soi.

On serait tenté d'imaginer que c'est le propre du maintien à domicile et que le placement en institution paralyse toute tentative de ce type. Mais les données recueillies résistent à cette grille de lecture. Une sélection peut s'effectuer de la part du résident, concernant ce qu'il peut et veut garder comme activités et relations, pour délaisser le reste. La toilette occupe ici une place centrale. Josée, 93 ans, précise :

Je suis complètement dépendante, je fais ma toilette seule, je suis obligée, mais quand je suis fatiguée, elles viennent m'aider. Je ne me sens pas capable de faire la cuisine, je ne me sens plus capable... je suis souvent malade et je vis dans la solitude.

Le fait d'avoir du personnel (parfois 24h/24) à disposition, pour veiller aux résidents, distribuer les médicaments, servir les repas, faire le ménage, s'occuper du linge et les aider à faire leur toilette... est souvent mis en avant par les résidents eux-mêmes et leurs familles comme un élément facilitant la prise décisionnelle. Si la surveillance et l'assistance sanitaire sont souvent les premiers services recherchés, quelques zones d'ombre viennent obscurcir le tableau. Ernestine nous explique :

Autant pour les aides ménagères, les infirmières ou le secrétariat, elles sont toujours là. Mais quand on est habitué à l'ancien personnel, il faut faire attention, on doit s'adapter à chaque fois. J'ai moins de liens avec elles, je suis moins libre que je ne l'étais. Une simple plaisanterie amicale je ne la ferai pas, je suis moins à l'aise. Je suis sur la réserve... 
Nuançons le propos : cette difficulté n'est pas réservée aux personnes vivant en institution. Hélène, 85 ans, qui vit toujours à son domicile, nous révèle: "Cette semaine, j'ai eu trois aideménagères différentes. Trois! Comment voulez-vous qu'il y ait une relation de confiance? Je n'arrive pas bien à retenir leurs noms, leurs histoires et je ne sais jamais quand je vais les revoir. C'est jamais les mêmes...»

\section{Conclusion}

Dans le cadre de cet article nous nous sommes proposés de rendre compte des activités des aînés et des pratiques professionnelles du personnel impliqué dans son accompagnement sous l'angle de la théorie de la déprise. Il s'est agi alors pour nous de développer une réflexion située au croisement des savoirs pratiques, de terrain et de la recherche, en lien avec cette même théorie, en nous focalisant d'une part, sur les conditions de vie des personnes dites âgées, prises en charge dans des foyers logement non médicalisés ou vivant en domicile et, d'autre part, sur les mécanismes décisionnels situés à l'origine de leur placement en institution.

Trois angles d'approche ont été retenus: le deuil de soi, le sentiment de solitude et l'accompagnement. Du côté des personnes les plus âgées, nous nous sommes efforcés de rendre compte des formes de déprise observées tout au long de notre pratique professionnelle insistant sur les leviers de prise, le contrôle de pouvoir, la dynamique de rebonds et de réorganisation de la vie, avec des stratégies d'adaptation dans (et en dehors de) l'espace de l'institution. Du côté des professionnels des soins et dans une perspective interactionniste, nous avons cherché à faire état de l'intérêt que représente cette théorie pour les professionnels (et par conséquent pour les personnes aidées), tout en explorant ses limites et les enjeux professionnels qui l'animent. 